\title{
Effect of High Voltage Impulses on Surface Discharge at the Oil-Paper Interface
}

\author{
R. Clemence Kiiza, M. Ghaffarian Niasar, R. Nikjoo, X. Wang and H. Edin \\ KTH Royal Institute of Technology \\ School of Electrical Engineering \\ Teknikringen 33 \\ SE-100 44 Stockholm \\ Sweden
}

\begin{abstract}
High voltage transients in electrical power systems are mainly caused by lightning strikes and switching operations. The high voltage transients above the basic insulation level (BIL) would degrade the electrical insulation of the equipment.

In this work, the effect of high voltage impulses on the trend of the surface discharge at the oil-paper interface was investigated. The partial discharge patterns were recorded by the Insulation Condition Monitoring (ICM) system. The HV impulses were generated by a standard $1.2 / 50 \mu \mathrm{s} \mathrm{HV}$ impulse generator. The measurement results presented in this paper seem to show that surface discharge would cause complete breakdown (continuous flashovers) at the oil-paper interface much faster when the test sample is first aged by $\mathrm{HV}$ impulses compared to when it is unaged.
\end{abstract}

\section{Introduction}

The electrical insulation system is one of the most important parts in HV equipment. One of the essential $\mathrm{HV}$ equipment in electrical power system is the power transformer bushing. There would be problems in the transformer bushings when in operation such as partial discharges in cavities or surface discharge at the oilpaper interface. The detail information about the problems in the condenser bushings can be found in [1]. This work has concentrated on surface discharge at the oil-paper interface.

The studies to investigate the surface discharge at the oil-solid interface were done in [2-8]. Majority of these studies focused at the oil-pressboard interface. The motivation to these studies is driven by the fact that pressboards are frequently used as one of the insulation materials inside the power transformer to separate windings and discs within the winding. Due to complication in transformer design there would be places where the electric field stress component is parallel, and if the parallel electric field stress reaches the discharge inception stress can cause surface discharge [2]. Impurities in oil which are likely to jam into the oil wedge placed between the windings and the pressboard block would also be the source of the surface discharge on the pressboards [3]. Little studies focused directly to paper insulation, which is one of the commonly used solid insulation in power transformer and its bushings. Specifically, in power transformer bushing there could be surface discharge at the oil-paper interface initiated from overstressed edges of metal foils.

This work compares the trends of surface discharges at the oil-paper interface when oil-impregnated paper used in power transformer bushings are unaged and when they are first aged by HV impulses. The investigation was done on the simple test set-up modeling the surface discharges at the oil-paper interface.

\section{Experimental setup}

In this section, test samples and measuring instruments used in this work are described.

\subsection{Test cell}

A schematic diagram of a test cell used in the experiments is shown in Fig.1. It consisted of Munksjö Thermo70 paper layers used in power transformer bushings, rod and plane brass electrodes.

The relative permittivity of unimpregnated paper as calculated from the measured capacitance at $50 \mathrm{~Hz}$ was about 2.1. The capacitance was measured by the HP 4284A LCR meter. The paper layers were impregnated with Nytro10XN transformer oil. The impregnation followed the procedures as in [9]. Four test samples each with 20 oil-impregnated paper layers $(0.1 \mathrm{~mm}$ thickness per layer) were used in experiments. The relative permittivity of each of the four test samples at $50 \mathrm{~Hz}$ was about 3.5 .

The test cell was set in such a way that there would be progressive surface discharges at the oil-paper interface on one side of the electrodes. To achieve this as shown in Fig.1, the HV brass electrode had sharp edges on the sides next to the oil-impregnated paper surface in order to initiate surface discharge easily. In additional to that the oil-impregnated paper layers did not cover 
completely a part of the bottom brass (ground) electrode.

From

$$
E_{t}=\frac{U}{L}
$$

where, $E_{t}$ is the average tangential electric field stress at the oil-paper interface, $L$ is the length of the surface of the insulation system from the ground electrode to the $\mathrm{HV}$ electrode, and $U$ is the applied voltage at the $\mathrm{HV}$ electrode; the geometry configuration would result in high average tangential electric field stress $\left(E_{t}\right)$ on the side with shorter $L$ as compared to the one with longer $L$.

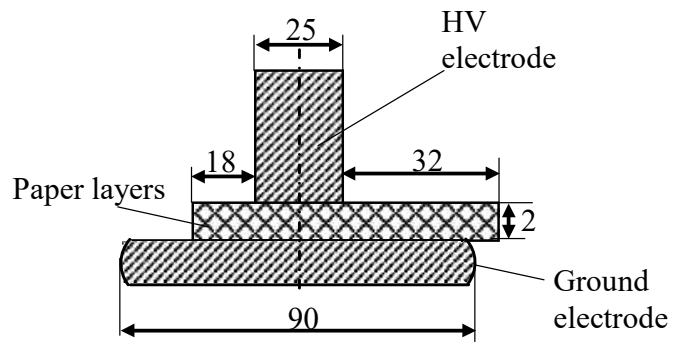

Fig. 1 - A test cell for investigation of surface discharge at the oil-paper interface. The figure is not drawn according to scale. All dimensions are in $\mathrm{mm}$.

During partial discharge (PD) experiments, the test cell was completely immersed in Nytro 10XN transformer oil. Due to difference in relative permittivity of the transformer oil and oil-impregnated paper, the electric field stress will concentrate in the transformer oil. When the magnitude of tangential electric field stress at the oil-paper interface reaches a critical threshold value, surface discharge would occur.

\subsection{Phase resolved partial discharges measuring system}

The schematic diagram of phase resolved partial discharges (PRPD) measuring system is shown in Fig. 2; where ZD is the detection impedance and RPA1 is the preamplifier. The detail description of this system can be found in [9].

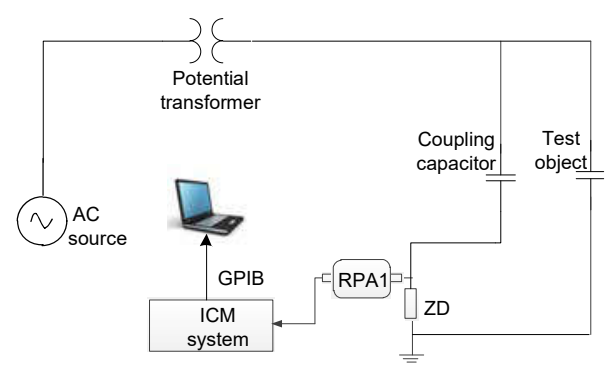

Fig. 2 - Schematic diagram of PRPD measuring system.

\section{Experimental procedures}

In order to accomplish the main objective of this work, which is a comparison of the trends of surface discharge at the oil-paper interface when it is initially unaged and when it is first aged by $\mathrm{HV}$ impulses; two sets of experiments were performed. The first set of experiments was performed on two test samples, namely sample 1 and sample 2 to investigate the effect of elevated $\mathrm{AC}$ voltage to the surface discharge at unaged oil-paper interface. Each of these test samples was subjected to an AC stress of $17 \mathrm{kV} / \mathrm{mm}$. The total experimental time was about $2 \frac{1}{2}$ hours for sample 1 , and 4 hours for sample 2 .

In the second set of experiments, the other two test samples (sample 3 and sample 4) were used in the investigation of the trends of surface discharge at aged oil-paper interface. Each of the two test samples was first subjected to $10 \mathrm{HV}$ impulses $(1.2 / 50 \mu \mathrm{s})$ of peak stress of $45 \mathrm{kV} / \mathrm{mm}$. Then, the test sample was connected to PRPD measurement setup to measure surface discharge at oil-paper interface. The applied AC stress was also $17 \mathrm{kV} / \mathrm{mm}$. Each of the experiments for sample 3 and sample 4 ended after observing at least 6 continuous flashovers within the same acquisition period of 5 minutes. The PRPD pattern was recorded every 5 minutes up to the end of each experiment.

\section{Experimental results and discussion}

In this section some of phase resolved partial discharge patterns (PRPD) are presented. Also, the development of paper surface tracking has been discussed.

\subsection{Phase Resolved PD Patterns of surface discharge at an unaged and aged oil-paper interface}

During the surface discharge activities, the complete discharges (flashovers) were observed in some of the acquisition periods. The PRPD patterns of each of the test samples for some of the acquisition periods before and after flashovers are shown in Fig. 3.

As it can be seen from Fig. 3, the PRPD patterns for the acquisition period with and without flashovers seem to differ for all four test samples. The PD pulses in the acquisition period in which flashover(s) happened appeared in a wide interval of the phase angles of the applied $50 \mathrm{~Hz}$ AC voltage, as compared to those with no flashovers. This kind of pattern was also observed in [4].

\subsection{Development of the paper surface tracking}

The photos of the test samples after experiments as taken by a digital camera are as shown in Fig. 4. All test samples were carbonized by surface discharge activities 


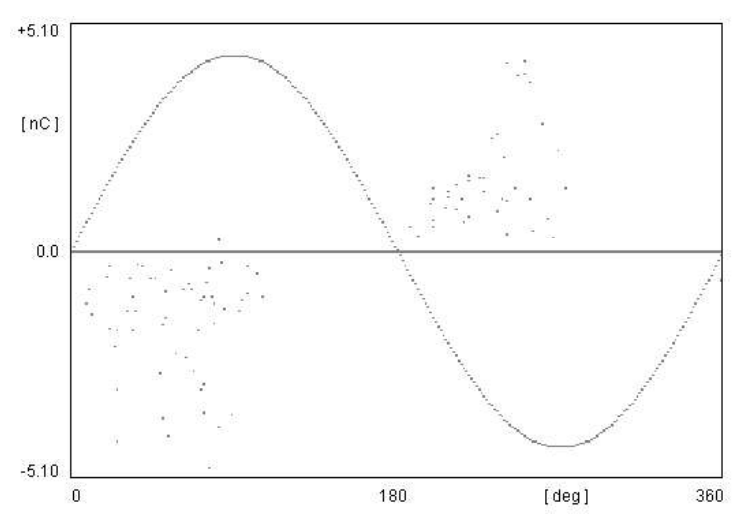

(a) Sample 1: PRPD pattern after 0 minute (no flashover).

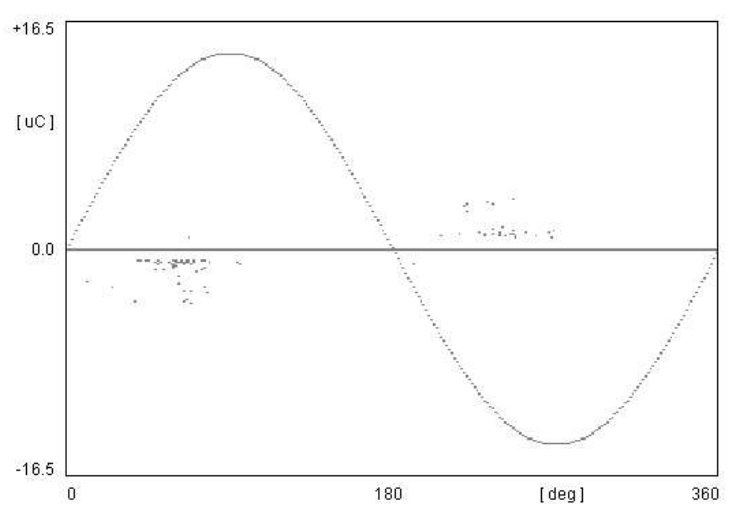

(c) Sample 2: PRPD pattern after 40 minutes (no flashover).

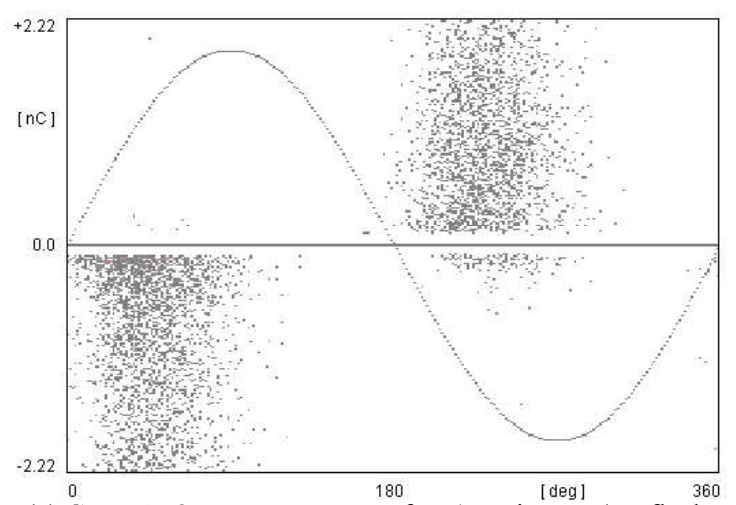

(e) Sample 3: PRPD pattern after 15 minutes (no flashover).

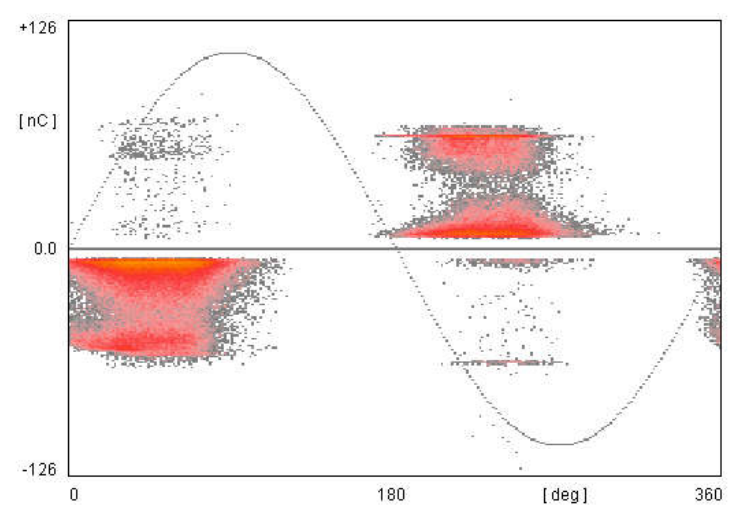

(g) Sample 4: PRPD pattern after 95 minutes (no flashover).

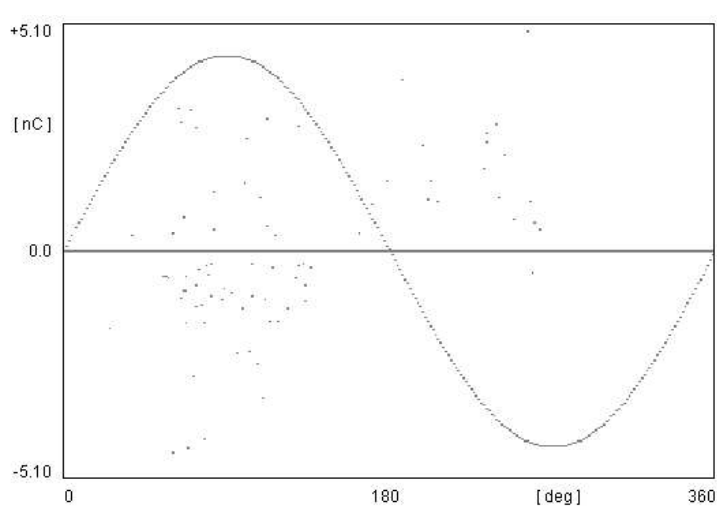

(b) Sample 1: PRPD pattern after 5 minutes (one flashover).

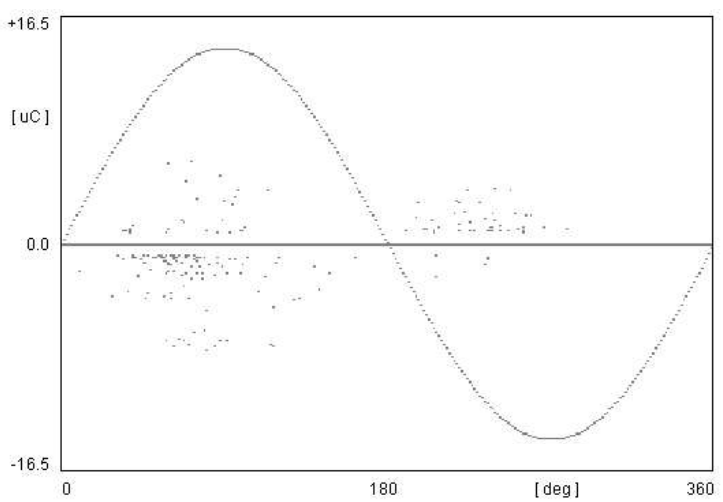

(d) Sample 2: PRPD pattern after 45 minutes (one flashover).

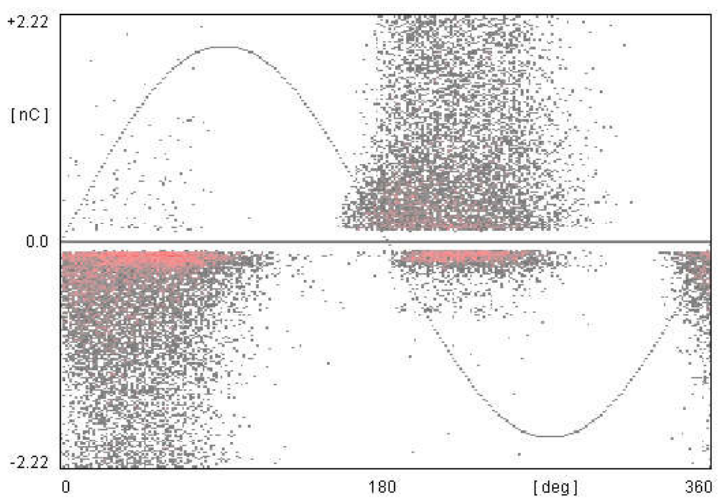

(f) Sample 3: PRPD pattern after 20 minutes (one flashover).

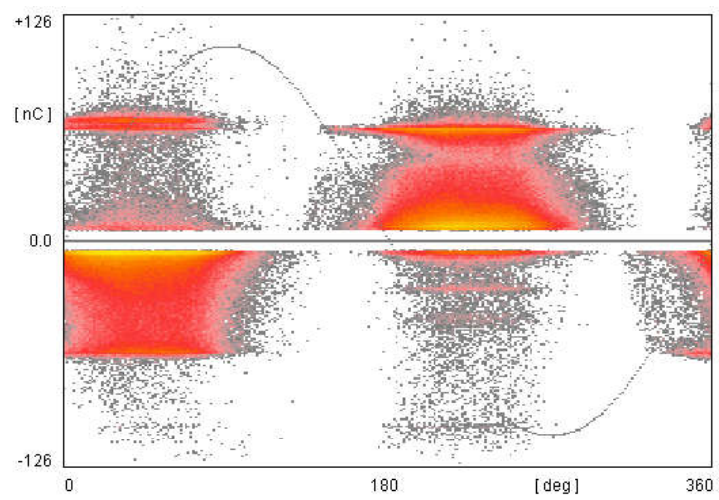

(h) Sample 4: PRPD pattern after 105 minutes (11 flashovers).

Fig. 3 - Phase Resolved PD patterns for initially unaged oil-paper interfaces (sample 1 and sample 2), and initially aged oil-paper interfaces (sample 3 and sample 4) for the acquisition periods without and with flashover(s). The acquisition time for each PRPD pattern was 5 minutes. 
around the edges of HV electrode and on the side of the bottom electrode which was not covered completely by paper layers. The HV impulses caused the cracks on the paper surface as seen in Figs. 4(c) and 4(d). The elevated AC stress on the test samples initially aged by $10 \mathrm{HV}$ impulses (sample 3 and sample 4) caused continuous flashovers, but did not cause continuous flashovers to initially unaged test samples.

The difference may be due to the cracks on the paper surface caused by HV impulses. It is reported in [5] that surface cracks is one of the factors which can affect the surface discharge activity. These cracks when either filled or unfilled with oil have relative permittivity less than relative permittivity of oil-impregnated paper. Thus, paper surface containing the cracks would result in non-uniform tangential electric field distribution, and the magnitude of tangential electric field stress in the cracks would be higher than in the rest part of the paper surface. The surface tracking is then likely to grow fast at the edges of the cracks.

The results also seem to show that the complete discharges (flashovers) would not necessarily cause carbonization of paper, because all $10 \mathrm{HV}$ impulses caused flashovers on the surface of paper layers, but did not cause carbonization. Carbonization occurs in dry parts of the cellulose insulation [6]. It would be preceded by white marks, which is usually associated with gas bubbles. The white marks can be seen especially in Figs. 4(b), 4(c) and 4(d). The gas bubbles coming from paper layers can be considered as the sign of dryness of the paper [5].

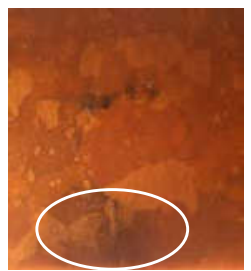

(a) Sample 1

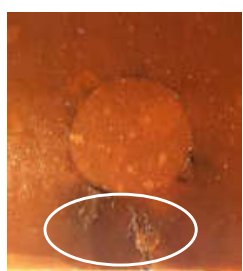

(c) Sample 3

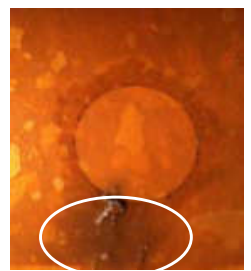

(b) Sample 2

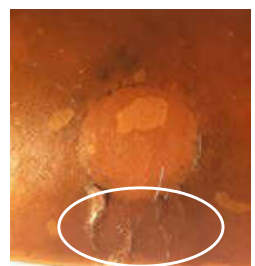

(d) Sample 4
Fig. 4 - Test samples after subjected to surface discharge activities at elevated AC stress: (a) and (b) are samples which were initially unaged, and (c) and (d) are samples which were initially aged by $10 \mathrm{HV}$ impulses.

\section{Conclusions}

This work compared the trends of surface discharge at the oil-paper interface when oil-impregnated paper layers used in power transformer bushings were initially unaged and when they were first aged by HV impulses. As it was intended the developed model caused the development of the paper surface tracking towards one side of the electrodes.

The results show that the flashovers caused by HV impulses would damage the surface of the paper layers on the HV electrode side resulting in cracks on the surface. The electric fields would concentrate on the cracks and speed up the surface tracking process. For instance the complete breakdown (continuous flashovers) of one of the test samples initially aged by HV impulses happened after about $1 \frac{1}{4}$ hours of surface discharge activity, but for the case of initially unaged test sample there was no complete breakdown (continuous flashovers) even after 4 hours of surface discharge activity.

\section{Acknowledgment}

The project was supported by the Royal Institute of Technology (KTH) and Dar es Salaam Institute of Technology (DIT), and is gratefully acknowledged.

\section{References}

[1] K. Ellis, Bushings for power transformers: A handbook for power engineers, Bloomington, Electric Connection, Inc., 2011.

[2] M.Ghaffarian Niasar, R. Clemence, X.Wang, R.Nikjoo and H.Edin, Effect of temperature on surface discharge in oil, IEEE CEIDP, 2012, pp. 96- 99 .

[3] Y. Cheng, C.Li and Y.Miao, The relationship between surface partial discharge and carbon trace in oil-impregnated paper insulation, IEEE CEIDP, 2009, pp. 96 - 99.

[4] Y.Cheng, E.Gockenbach, C.Eichler and C.Li, The partial discharge phenomena on the surface of oil impregnated paper with parallel electric field, IEEE Annual Report CEIDP, 2010.

[5] P.M.Mitchinson, P.L.Lewin and B.D. Strawbridge, Tracking and surface discharge at the oilpressboard interface, IEEE DEIS Feature Article, 2010, Vol.26, No.2, pp.35-41.

[6] H.Zainuddin, P.M.Mitchinson and P.L.Lewin, Investigation on the surface discharge phenomenon at the oil-pressboard interface, IEEE Int. Conf. on Dielectr. Liquids, 2011.

[7] H. Wang, C. Li and H. He, Influence of temperature to developing processes of surface discharges in oil-paper insulation, IEEE Int. Symp. on Electr. Insul. (ISEI), 2010.

[8] J.Li, W. Si, X.Yao and Y.Li, Partial discharge characteristics over differently aged oil/pressbord interfaces, IEEE Trans. on Dielectr. and Electr. Insul., 2009, Vol.16, No.6, pp.1640-1647.

[9] R. Clemence Kiiza, M. Ghaffarian Niasar, R. Nikjoo, X. Wang and H. Edin, Comparison of phase resolved partial discharge patterns in smalltest samples, bushing specimen and aged transformer bushing, IEEE CEIDP, 2012, pp. 88 91. 